\title{
Investigação da propagação de ondas de gravidade no terremoto do PERÚ em 26 novembro de 2015 as 05:45:18 (UTC) observadas nas estações de GPS na Amazônia.
}

\author{
${ }^{1}$ FERREIRA, A., 'LIMA, N., ${ }^{1}$ SILVA, R*., ${ }^{1}$ GÓES, K., ${ }^{1} F A R I A S, T$. \\ (1) Centro Universitário Luterano de Manaus - CEULM/ULBRA \\ Av. Carlos Drummond de Andrade, 1460. Conjunto Atílio Andreazza. Bairro Japiim II. \\ Manaus-AM/Brasil. CEP. 69077-730. Tel. (92) 3616-9800. \\ (e-mail:newtonulbra@gmail.com)
}

Copyright 2016, SBGf - Sociedade Brasileira de Geofísica

Este texto foi preparado para a apresentação no VII Simpósio Brasileiro de Geofísica, Ouro Preto, 25 a 27 de outubro de 2016. Seu conteúdo foi revisado pelo Comitê Técnico do VII SimBGf, mas não necessariamente representa a opinião da SBGf ou de seus associados. É proibida a reprodução total ou parcial deste material para propósitos comerciais sem prévia autorização da SBGf.

\section{Resumo}

Este trabalho faz uma breve observação de propagação de ondas de gravidade na ionosfera (Região F) sobre a cidade de Manaus - Brasil, durante um evento de terremoto no Perú Central de intensidade Mw 7.6 em 24 de Novembro de 2015, com uso de GPS Ionosférico localizado no Centro Universitário Luterano de Manaus (CEULM/ULBRA - Brasil), em comparação com outras estações de GPS na Amazônia, que registraram e identificaram a instabilidade do plasma ionosférico durante a passagem de ondas de gravidade após o terremoto.

\section{Introdução}

Durante a guerra fria em 30 de outubro de 1961, uma explosão nuclear no arquipélago de Novaya Zemlya (Nova Zembla), ao norte da Rússia, causou uma enorme perturbação na ionosfera, fazendo variar freqüência de rádio, também em 1964 um terremoto no Alasca produziu o mesmo efeito em alta atmosfera, ou seja, propagação de ondas de gravidade acústica acima de $100 \mathrm{~km}$ de altitude (ROW, 1967). Minster (1996) relatou que um terremoto no Perú em 17 janeiro de 1994, de intensidade $\mathrm{Mw}=6.7$, que induziu ondas de gravidade acústica que se acoplaram, fazendo variar o conteúdo eletrônico total da ionosfera que foram registrados com a utilização de GPS. Hegai et al., (2006), investigaram perturbações na região $\mathrm{F} 2$ em média latitude durante a noite na ionosfera que fora produzida por ondas internas gravidade geradas antes de um forte terremoto através de aquecimento Joule devido ao campo elétrico de sismogênicos de curta duração, na Região $D$, da ionosfera. Toda esta teoria se assenta sobre um artigo de C. O. Hines de 1965, com 6 páginas intitulado "Ondas de Gravidade Atmosférica: Uma nova ferramenta para teoria de onda" (Journal Radio Science Canada) em (Nappo, 2002).

$\mathrm{Na}$ geociência, Sauli, et al. (2007) menciona que um dos trabalhos pioneiro do estudo de ondas de gravidade foi realizado por Hines (1960) e posteriormente os trabalhos de Herkrider (1964) e Row (1967) relacionaram perturbações do tipo OG com explosões e terremotos. E mais recentemente, utilizando técnicas modernas (GPS, gravímetros e sismógrafos), várias investigações indicam a propagação de $\mathrm{OG}$ após acontecimentos de terremotos, explosões naturais ou artificiais e erupções vulcânicas Lognonné et al.(2007), Tolstoy, et al. (1970), Jung, et al. (2006), Molchanov, et al. (2004), Erickson, et al.(2008).

\section{Metodologia}

\section{Aquisição dos dados}

O instrumento de aquisição de dados é um GPS Ionosférico (NovAtel - Nov4004GSV instalado no Laboratório da Ionosfera do Centro Universitário Luterano de Manaus - CEULM/ULBRA(-3,1082690;

$59,9746552)$ de dupla frequência ( $L 1$ e $L 2$, para minimizar o efeito da refração) e antena (NOV702rev.4) apropriada para minimizar o efeito de multicaminho (Fig. 01). Ligado diretamente a um PC e a rede internet no site LISN (LISN - Low-Latitude lonospheric Sensor Network) em (http://lisn.igp.gob.pe/) com operação contínua.

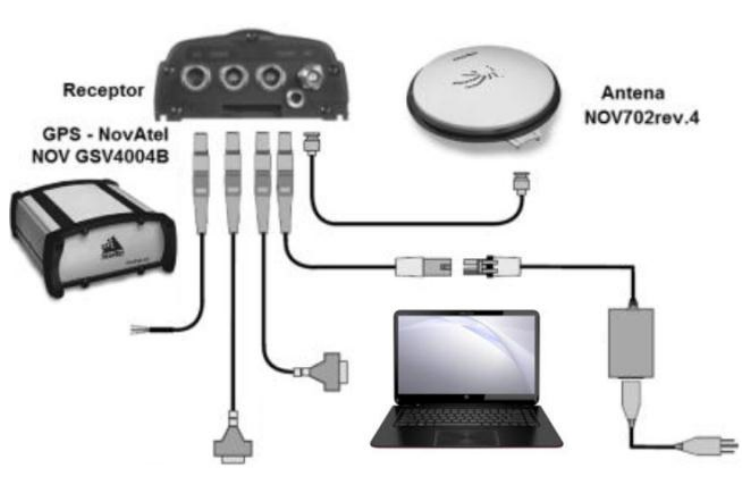

Fig.01: Escopo da Estação GPS Manaus (CEULM/ULBRA-UNIVAP) 


\section{O Método}

Através dos dados do GPS, gráficos de VTEC (Vertical Total Electron Content) e $\left(\mathrm{S}_{4}\right)$ Cintilação são criados para mostrar o estado do plasma ionosférico durante as varreduras dos satélites da rede GPS sobre a cidade de Manaus-Brasil, que são armazenados em arquivos diários e disponibilizados na rede LISN. A rede que é inserido os dados da Estação Manaus é chamada de UNIVAP.

\section{Resultados e Discussão}

$\mathrm{Na}$ terça feira do dia 24 de novembro de 2015, no centro do Perú (norte do mosaico da América do Sul) um forte terremoto de intensidade $\mathrm{Mw} 7.6\left(10.05^{\circ} \mathrm{S} ; 71.02^{0} \mathrm{~W}\right)$ com profundidade de $611 \mathrm{~km}$, as 22:50:53 UTC (17:50:53 local), (Fig. 02), atingiu áreas adjacentes ao epicentro, chegando a ser percebido na cidade de Manaus-Brasil, conseqüentemente degradando 0 sinal GPS, aqui registrados em suas respectivas estações.
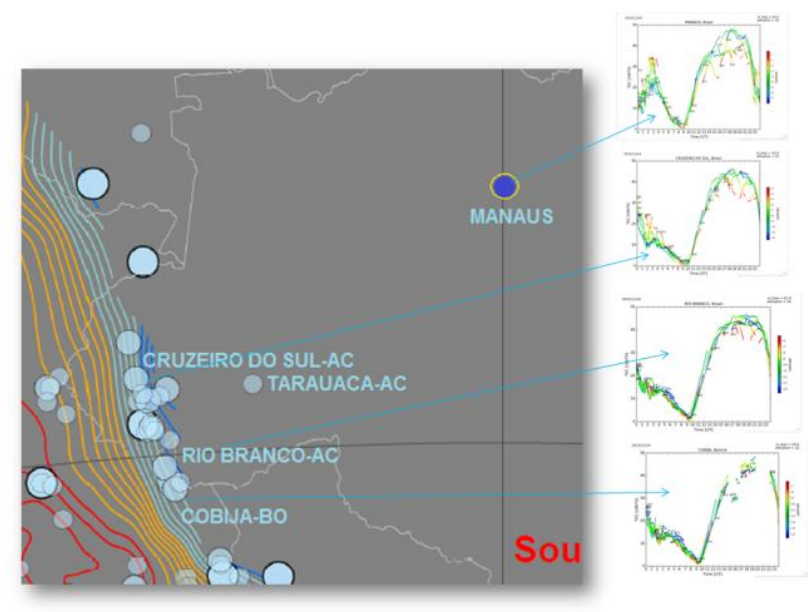

Fig. 02: Região do epicentro do evento e adjacentes na Amazônia, no dia do evento. (LISN; USGS, 2015).

Durante o evento as estações GPS em toda a Amazônia que foram consultadas em três dias, ou seja, um dia antes do evento, um durante e outro depois (Fig. 03), mostraram o conteúdo total da ionosfera (Região F), e certificaram a existência de passagem de ondas de gravidade sobre 0 alvo evidenciado pelos gráficos de VTEC (Vertical Total Electron Content), com degradação do sinal GNSS (Global Navigation Satelite System), na aquisição os dados.
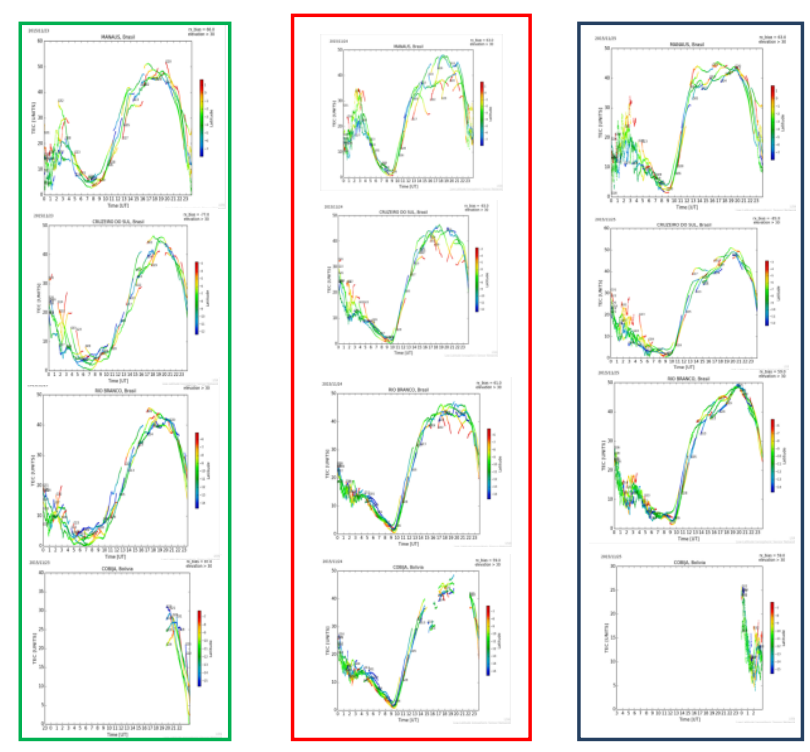

Fig. 03: Painel de VTEC nas Estações da Amazônia explicitado através de colunas; sendo a primeira para um dia antes do evento e segunda para o dia do evento e a terceira um dia após o evento, para as seguintes localidades de cima para baixo das colunas (Manaus/Amazonas- Cruzeiro do Sul/ Acre -Rio Branco/Roraima-(Brasil); Cobija (Bolívia)). LISN, 2015.

\section{Conclusões}

É possível observar evento de terremoto através de ondas de gravidade na região $\mathrm{F}$ da ionosfera com auxílio de GPS, segundo autores citados, na região equatorial da Amazônia Central.

\section{Agradecimentos}

Os autores são gratos ao Edital 030/2013 FAPEAM, a LISN, ao USGS, SIGAS (IBGE).

\section{Referências}

ERICKSON, B., BIRNIR, B., LAVALLÉE, D. 2008. A model for aperiodicity in earthquakes, Nonlin. Processes in Geophys., 15, 1-12.

HARKRIDER, DAVID G. (196). Part I. Propagation of acoustical gravity waves from an explosive source in the atmosphere. Part II. Rayleigh and Love waves from sources in a multilayered elastic half-space, CalTech California - USA. Dissertation - PhD in Geological and Planetary Sciences, 285p. 
HEGAI, V. V.; LIU, J. Y.; KIM, V.P. (2006) The ionospheric effect of atmospheric gravity waves excited prior to strong earthquake. Advances in Space Research 37-653-659. Disponível em: <HTTP://www.researchgate. Net/publication/222556220>.

JUNG, T. K., LIU, J. Y. TSAI, H. F., HUANG, B. S., LIN, C. H., YU, S. B., YEH, Y. S. (2006). Ionospheric disturbances triggered by the Mw 7.6 earthquake off the Coast of El Salvador on 13 January 2001. Terr. Atmos. Ocean. Sci., 17, 345-351.

LOGNONNÉ, P., GARCIA, R., CRESPON, F., OCCHIPINTI, G., KHERANI, A., LAMBIN-ARTRU, J. (2007). Seismic waves in the atmosphere. Euro Physics News, 37 (4), 11-14.

MINSTER, J. BERNARD. (1995) GPS detection of ionospheric perturbations following the January 17, 1994, Northridge Earthquake. DOI: 10.1029/95GL00168. Geophysical Research Letters. Vol. 22. Pages 10451048, May 1995.

MOLCHANOV, O., FEDOROV, E., SCHEKOTOV, A., GORDEEV, E., SURKOV, V., ROZHNOI, A., ANDREEVSKY, S., IUDIN, D., YUNGA, S., LUTIKOV, A., HAYAKAWA, M., BIAGI, P. F. 2004. Lithosphereatmosphere-ionosphere coupling as governing mechanism for preseismic short-term events in atmosphere and ionosphere. Natural Hazards and Earth System Sciences. 92, 757-767.

NAPPO, C. J. 2002. An Introduction to Atmospheric Gravity Waves, Academic Press, An imprint of Elsevier Science, (USA), International Geophysics series, v. 85, $276 p$.

ROW, RONALD V. (1967) Acoustic-gravity waves in the upper atmosphere due to a nuclear detonation and an earthquake. DOI: 10.1029/JZ072i005p01599. Journal of Geophysical Research. Vol. 72. No. 5. March,1967.

SAULI, P., and BOSKA, J. (2001). Tropospheric events and possible related gravity wave activity effects on the ionosphere, J. Atmos. Sol. Terr. Phys., 63, 945- 950.

TOLSTOY, I., HERRON, T. J. 1970. Atmospheric gravity waves from nuclear explosions. Journal the Atmospheric Sciences, 27, 55-61. 